\title{
Functional interconnections of HY1 with MYC2 and HY5 in Arabidopsis seedling development
}

\author{
Babu Rajendra V Prasad ${ }^{1}$, Selva V Kumar ${ }^{1}$, Ashis Nandi ${ }^{2}$ and Sudip Chattopadhyay ${ }^{3 *}$
}

\begin{abstract}
Arabidopsis seedling development is controlled by many regulatory genes involved in multiple signaling pathways. The functional relationships of these genes working in multiple signaling cascades have started to be unraveled. Arabidopsis HY1/HO1 is a rate-limiting enzyme involved in biosynthesis of phytochrome chromophore. HY5 (a bZIP protein) promotes photomorphogenesis, however ZBF1/MYC2 (a bHLH protein) works as a negative regulator of photomorphogenic growth and light regulated gene expression. Further, MYC2 and HY1 have been shown to play important roles in jasmonic acid (JA) signaling pathways. Here, we show the genetic interactions of HY1 with two key transcription factor genes of light signaling, HY5 and MYC2, in Arabidopsis seedling development. Our studies reveal that although HY1 acts in an additive manner with HY5, it is epistatic to MYC2 in light-mediated seedling growth and gene expression. This study further demonstrates that HY1 additively or synergistically functions with HY5, however it works upstream to MYC2 in JA signaling pathways. Taken together, this study demonstrates the functional interrelations of HY1, MYC2 and HY5 in light and JA signaling pathways.
\end{abstract}

\section{Background}

Light is one of the most important environmental factors for plant growth and development throughout its life cycle $[1,2]$. Plants have evolved with multiple photoreceptor-systems to monitor the surrounding light quality, quantity, and direction. In Arabidopsis, these photoreceptors include the blue/UV-A light absorbing cryptochromes (CRY1 to CRY3) and phototropins (PHOT1 and PHOT2); the red/far-red light absorbing phytochromes (phy: phyA to phyE) [3-7]. Arabidopsis phytochromes form homo and hetero dimers with each other [8-10]. Formation of such heteromeric photoreceptors increases the potential complexity of R/FR light sensing and signaling mechanism in plants. Similarly, light induced activation of cryptochromes leads to possible autophosphorylation and dimerization [11]. Moreover, phytochromes and cryptochromes work together either by interaction with each other in a lightdependent and interdependent manner [12,13].

Arabidopsis seedlings exhibit two distinct developmental patterns, photomorphogenesis or skotomorphogenesis depending on the presence or absence of light, respectively [13-15]. Skotomorphogenesis is the strategy followed

\footnotetext{
* Correspondence: sudipchatto@yahoo.com

${ }^{3}$ Department of Biotechnology, National Institute of Technology, Mahatma

Gandhi Avenue, Durgapur 713209, West Bengal, India

Full list of author information is available at the end of the article
}

under dark conditions where Arabidopsis seedlings exhibit elongated hypocotyl, closed cotyledons with apical hooks; whereas in presence of light, photomorphogenesis is initiated, characterized by short hypocotyl with fully developed cotyledons. This developmental change from skotomorphogenesis to photomorphogenesis is carried out by different classes of photoreceptors, and characterised by a change in the expression of about one-third of genes in the Arabidopsis genome [16-18].

Genetic screen of Arabidopsis seedlings for developmental defects under light conditions have led to the identification of several transcription factors that either act as a positive or negative regulator downstream to specific photoreceptor or a set of photoreceptors [19-27]. Recently, a DNA-ligand binding screen has led to the identification of three Z-box binding factors, ZBF1/MYC2, ZBF2/GBF1 and ZBF3/CAM7 [28-33]. MYC2 is a bHLH transcription factor that acts downstream to cry1 and cry2 photoreceptors, and negatively regulates blue light-mediated photomorphogenic growth and blue and far red-light regulated gene expression [29]. MYC2 also functions as a transcriptional regulator for $\mathrm{ABA}$ and JA signaling pathways [29,34-37].

HY5 is one of the first known and most extensively studied bZIP transcription factor involved in promoting photomorphogenesis. Arabidopsis seedlings mutant for
C Biomed Central

(C) 2012 Prasad et al; licensee BioMed Central Ltd. This is an Open Access article distributed under the terms of the Creative Commons Attribution License (http://creativecommons.org/licenses/by/2.0), which permits unrestricted use, distribution, and reproduction in any medium, provided the original work is properly cited. 
HY5 exhibit elongated hypocotyl under various wavelengths of light, suggesting that functionally HY5 is downstream to multiple photoreceptors [19,38-40]. Further, hy5 mutant seedlings exhibit defects in root growth and reduction in chlorophyll and anthocyanin accumulation $[19,41]$. In addition, studies have shown the involvement of HY5 in both auxin and cytokinin signaling pathways [42-44], suggesting that HY5 might be a common intermediate in light and hormone signaling pathways. The chromatin immunoprecipitation (CHIP) assays have revealed that HY5 preferentially binds to more than 3000 chromosomal sites that were distributed in all the five chromosomes [45].

Arabidopsis $H Y 1$ encodes heme oxygenase (HO) that catalyses the committed step in the conversion of heme to biliveridin IX $\alpha(\mathrm{BV})$, which is further converted to photochromobilin through sequential steps and exported to cytoplasm where it binds to the newly synthesized apo-phys by an autocatalytic process to form functional holo-phytochrome. The HOs are encoded by a small gene family that includes $H Y 1, H O 2, H O 3$ and $H O 4$. Among all the four members of the $\mathrm{HO}$ family $H Y 1$ is highly expressed in almost all the tissues and plays a major role in synthesis of holo-phytochrome [46]. Seedlings mutant for $H Y 1$ exhibits elongated hypocotyl in red and far red light, and display defects in root development. Further, the light inducible genes such as $C A B, R B C S$ and $C H S$ are under-expressed in hy1 mutant background [47-49]. Recently, it has been reported that seedlings mutant for $H Y 1$ show elevated levels of JA and expression of JA-inducible defense genes [50].

In this study, in order to identify genes that might be working parallel to $H Y 5$, a genetic screen was set up using hy5-ks50 mutant lines through EMS mutagenesis. Gene cloning and genetic complementation analysis revealed that one of these mutants (enhancer of HY5: ehy5) contains a mutation in the HY1gene. We have investigated the interrelations of $H Y 1$ with two transcription factors, HY5 and MYC2, with respect to light-controlled Arabidopsis seedling development and JA responsiveness.

\section{Results}

\section{Mutations in EHY5 modulate HY5-controlled hypocotyl elongation}

HY5 is a key transcription factor in light signaling pathways that promote photomorphogenesis under a broad spectrum of light $[32,40]$. Although the hy5 mutant seedlings display elongated hypocotyl in light, the seedlings are not completely etiolated similar to dark grown seedlings. Therefore, there might be additional factors present that are involved in the promotion of photomorphogenesis under various wavelengths of light [39]. Recent studies have shown that CAM7/ZBF3 works in various wavelengths of light to promote photomorphogenic growth and light regulated gene expression. Further, HY5 and CAM7 work synergistically or additively in the promotion of photomorphogenesis [32].

In order to find additional factors that promote photomorphogenesis in concert with HY5, an extragenic enhancer screen was set up using hy5 mutant lines (hy5-ks50 mutant; 19) through EMS mutagenesis. Several double mutant lines that showed enhanced hypocotyl growth as compared to that of hy5 mutants were identified. One such mutant line, hy5 ehy5 (ehy5: enhancer of HY5) double mutant, was selected for further study. The segregated ehy5 line (obtained in F2 population from a back cross with wild type (Ws) was repeatedly backcrossed with wild type (Ws) to purify the mutation from any other back ground mutations.

The examination of seedling morphology revealed that neither ehy5 alone nor ehy5 hy5 double mutants exhibited any altered morphology in the dark (Figure 1A (a) and 1B). The characteristic long hypocotyl phenotype of hy5 in WL (white light) irradiation was further enhanced in ehy5 hy5 double mutants, exhibiting a super tall phenotype under various fluences of WL (Figure 1A (b) and 1C). To determine whether this reduced sensitivity of ehy 5 hy 5 phenotype is specific to a particular wavelength of light, the growth of 6-day-old ehy5 hy5 double mutant seedlings was tested in various wavelengths of light. As shown in Figure 1A (c) and 1D, ehy5 hy5 double mutants displayed further reduced sensitivity to far-red light (FR) as compared to ehy5 and hy5 single mutants, suggesting that EHY5 and HY5 additively control the hypocotyl growth in FR. On the other hand, hypocotyl length of ehy5 hy5 double mutants was found to be closer to either of the single mutants in red light (RL), suggesting that EHY5 and HY5 are likely to work in the same branched pathways in controlling the hypocotyl length in RL (Figure 1A (d) and 1E). The ehy5 mutants exhibited similar hypocotyl length to that of wild type in blue light (BL), and the hypocotyl length of ehy5 hy5 double mutants was similar to that of hy5 single mutants, suggesting that additional mutation in EHY5 does not affect the hy5 phenotype in BL (Figure 1A (e) and $1 \mathrm{~F}$ ).

\section{Map based cloning reveals that EHY5 encodes HY1}

To determine the genetic basis of EHY5 mutation, we followed map-based cloning strategy. The ehy5 mutants (WS) were genetically crossed to wild type ( $\mathrm{Col})$, and the resulting $F_{1}$ progeny showed wild type phenotype. $F_{1}$ plants were self-pollinated and since the ehy5 long hypocotyl phenotype is easy to score at the seedling stage, the EHY5 locus has served as a useful landmark for classical mapping. For fine mapping, the segregating $\mathrm{F}_{2}$ populations with the ehy5 phenotype were used for mapping with Simple Sequence Length Polymorphism (SSLP) and Cleaved and Amplified Polymorphic Sequence (CAPS) markers 


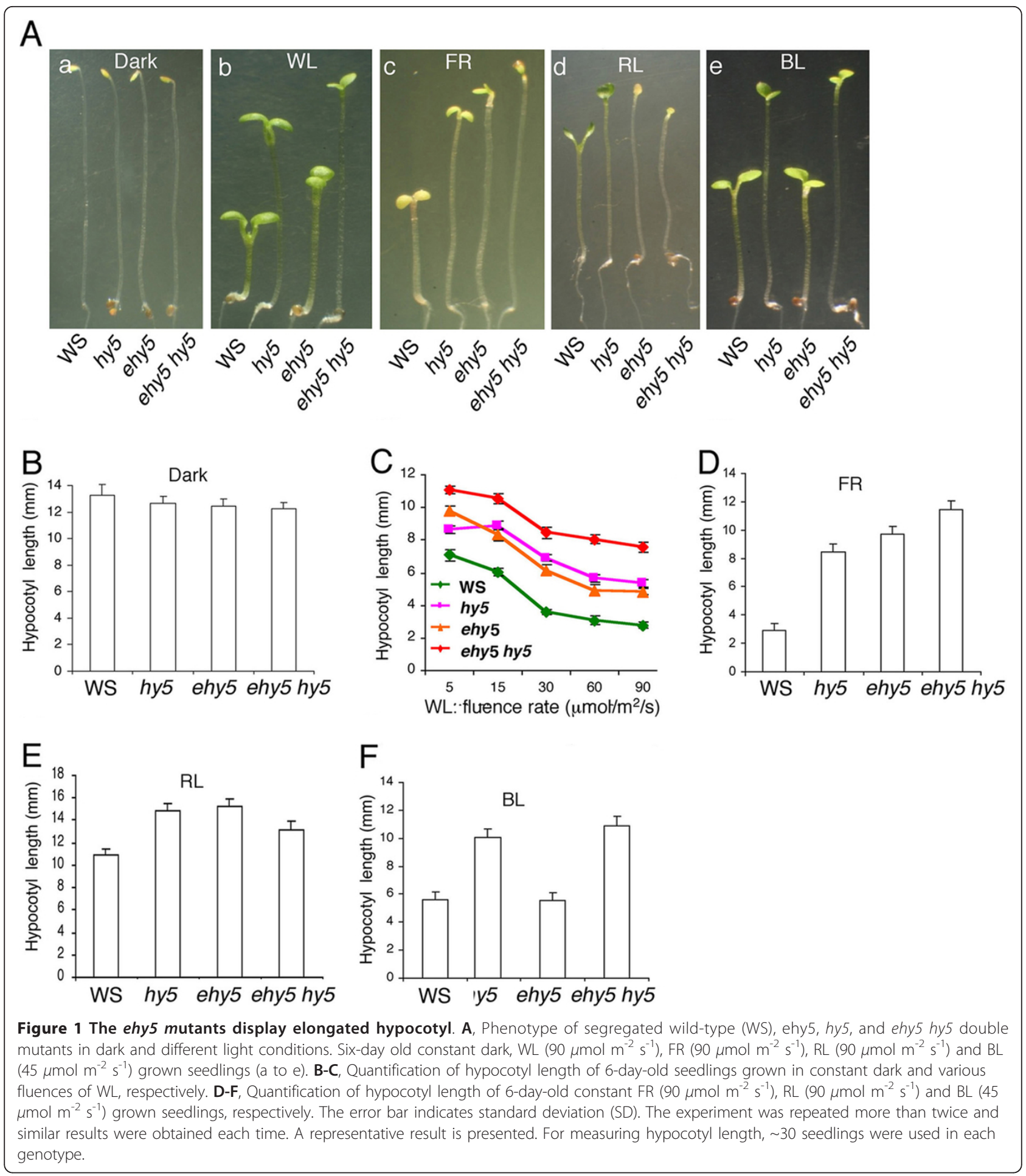

that we developed during this study and also that are available in the database at the Arabidopsis Information Resource (TAIR). Initially, the target locus was mapped between the markers ER and T20P8 on Chromosome 2 (Figure 2A). Further fine mapping with seven genetic markers delimited the target gene to a $20-\mathrm{Kb}$ region on the
F18A8 BAC clone. To further identify the exact position of the EMS mutation, we have sequenced the genomic DNA fragment of the 20-Kb region from the ehy5 background and compared with that of wild type (WS) genomic DNA sequence, which revealed that a single $C$ to $T$ nucleotide substitution in the first exon of the $H Y 1$ 


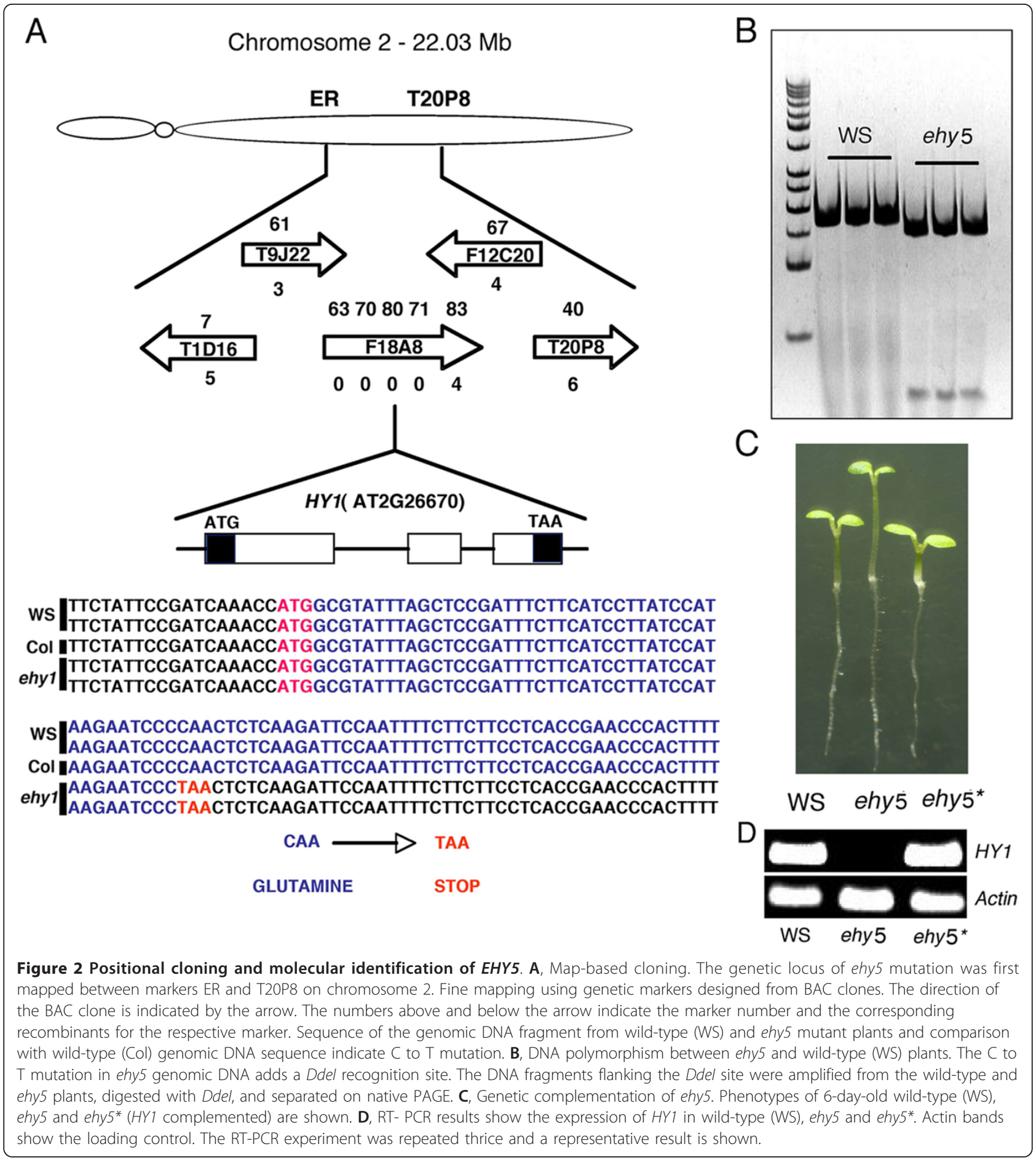

(AT2G26670) DNA leads to the conversion of Glutamine (CAA) to stop codon (TAA), resulting in the premature termination of the protein translation (Figure 2A). This EMS induced substitution in $H Y 1$ first exon introduces a DdeI recognition site adjacent to the mutation region. We developed a dCAPS marker to confirm the mutation in ehy5 (Figure 2B).
As a final step to establish that the EHY5 locus encodes $H Y 1$ transcript, we tested whether a wild type genomic fragment containing the entire $H Y 1$ gene could complement ehy5. Fragment containing entire $H Y 1$ coding region with its native promoter was introduced into ehy5mutant background. As shown in Figure 2C, ehy5 seedlings transformed with full length $H Y 1$ genomic DNA 
fragment exhibited wild-type phenotype. The positive transformants were confirmed by RT-PCR (Figure 2D). These results indicate that the ehy5 mutant is an allele of hy1 mutant, and henceforth we refer to ehy5 as hy1.

$H Y 1$ and $H Y 5$ additively regulate the expression of light regulated genes and accumulation of chlorophyll and anthocyanin during early seedling development

The loss-of-function mutants of HY5 display partial photomorphogenic growth at various wavelengths of light with reduced expression of light-regulated genes such as $C A B 1$ and $R B C S-1 A$. Similarly, hy1 also shows reduced accumulation of $C A B$ and $R B C S$ transcripts [51]. To examine how $H Y 5$ and $H Y 1$ genetically interact to regulate the expression of light inducible genes, we monitored the expression of $C A B 1$ and $R B C S-1 A$ by real time PCR. As shown in Figure 3A-B, the expression of $C A B 1$ and $R B C S-1 A$ was reduced in both hy 1 and $h y 5$ single mutants as compare to wild-type, and the accumulation of transcript was further reduced in hy1 hy5 double mutants compared to either of the single mutants. These results indicate that $H Y 1$ and $H Y 5$ act in an additive manner to regulate the expression of $C A B 1$ and $R B C S-1 A$ genes.

Earlier studies have shown that hy5 and hy 1 mutant seedlings display reduction in the accumulation of chlorophyll and anthocyanin. To determine the genetic interaction of $H Y 1$ and $H Y 5$ for chlorophyll and anthocyanin

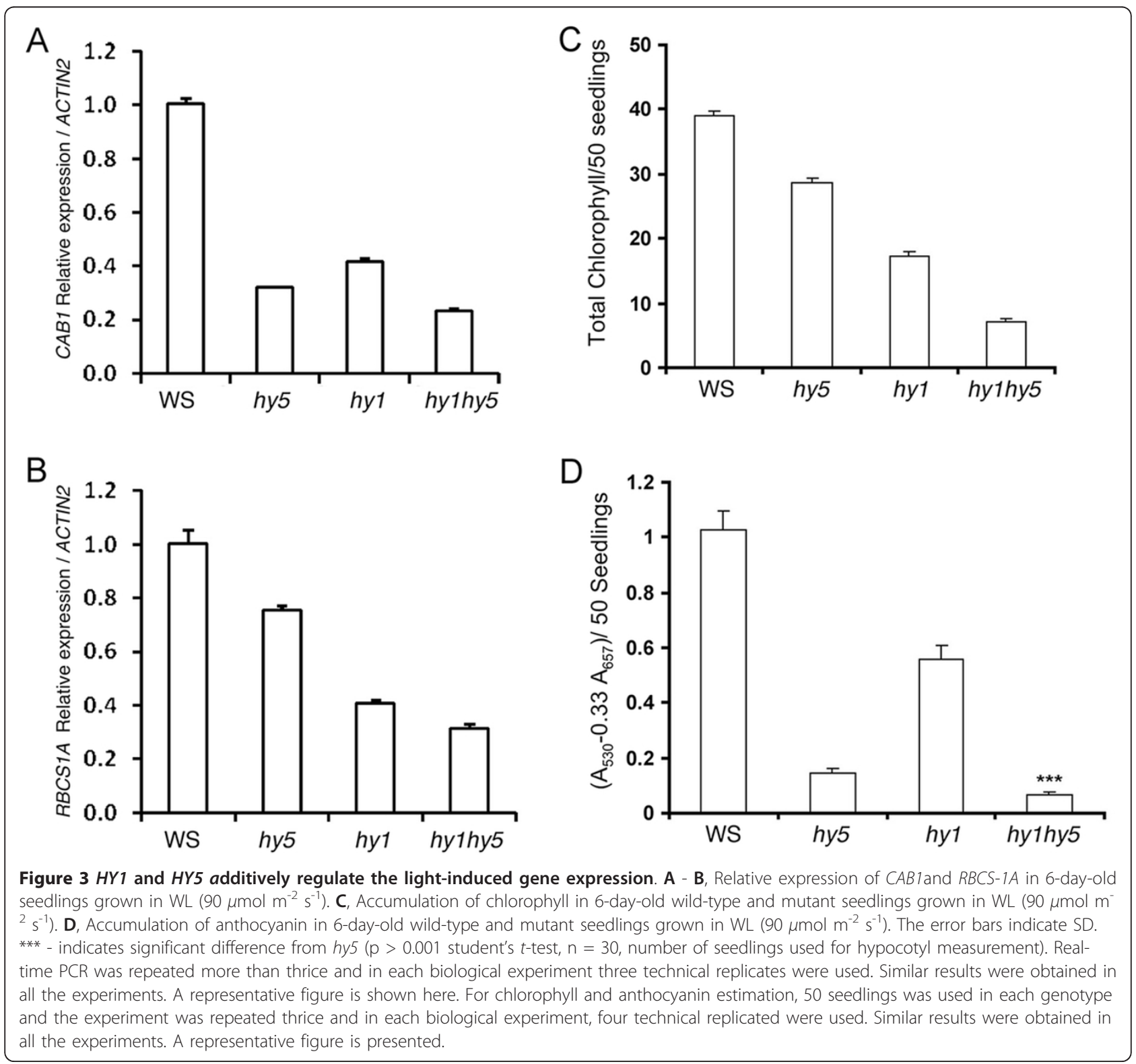


accumulation, chlorophyll and anthocyanin contents were estimated from six-day-old WL grown seedlings. As shown in Figure 3C and 3D, the hy1 hy5 double mutants showed less accumulation of chlorophyll and anthocyanin as compared to that of hy1 and hy5 single mutants, suggesting that HY1 and HY5 act in an additive manner to control the accumulation of chlorophyll and anthocyanin in WL.

\section{$H Y 1$ and $H Y 5$ work in an additive or synergistic manner to control JA responsiveness}

In the presence of jasmonic acid (JA), hy1 mutants have stunted root growth and expression of JA-inducible defence genes [50]. We asked whether mutation in HY5 can modulate the JA sensitiveness of hy1 mutants. To examine that, we grew the seedlings in the presence or absence of JA and examined the root growth. Although very little difference, if any, was observed between the wild type and hy1 mutants in the absence of JA (Figure 4A), $15 \mu \mathrm{M}$ JA caused root growth retardation in hy1 mutant seedlings as compared to the wild type (Figure 4B). The effect was more severe in hy1 hy5 double mutants (Figure 4B). These results suggest a synergistic function of HY1 and HY5 in JA-mediated root growth. To determine whether the expression of JA regulated genes is affected in hy1 hy5 double mutants, the transcript accumulation of JA-responsive marker gene VSP2 was determined [35,50]. The real time PCR analyses had shown that JA treatment induced the expression of VSP2 both in hy1 and hy5 mutants, and the level of expression was further increased in hy1 hy5 background (Figure 4C). These results indicate that HY1 and HY5 function in an additive manner to regulate the expression of VSP2 in response to JA.

\section{Additional mutation in $M Y C 2$ abolishes the hyper- sensitive responses of hy 1 to $\mathrm{JA}$}

MYC2, a bHLH transcription factor, acts as a negative regulator of blue light mediated photomorphogenic growth and cross talks with JA and ABA signaling pathways $[29,33,36,37,52,53]$. MYC2 positively regulates the expression of JA-responsive genes such as VSP2 by directly binding to the G-box motif present in the promoter of VSP2 [35,36]. Furthermore, atmyc2 mutant seedlings are insensitive to JA-induced inhibition of root growth. To investigate the interaction between $H Y 1$ and $M Y C 2$ with respect to JA-responses, we constructed hy1 atmyc2 double mutants through genetic crosses. The root growth of hy1 atmyc 2 double mutant plants was monitored in the absence or presence of $15 \mu \mathrm{M}$ JA. No significant difference in root length was observed among the mutants and wild type in the absence of JA. JA caused severe root growth retardation in wild-type and hy1 mutants, however the effect was drastically reduced in atmyc 2 and hy1 atmyc2 mutant plants (Figure 5A and
$5 \mathrm{~B})$. These results indicate that MYC2 works downstream to HY1 in JA-mediated inhibition of root growth. We then examined the expression of one of the JA-inducible marker genes VSP2 by real time PCR in various mutant backgrounds. As shown in Figure $5 \mathrm{C}$, whereas there was very little expression of VSP2 in the absence of JA, the expression of VSP2 was increased in the presence of JA in wild-type and hy1 mutant plants. Further, the hy1 mutants showed significantly higher level of accumulation of VSP2 transcript as compared to wild-type background. However, the expression of VSP2 was less in atmyc 2 plants, as expected from its less sensitiveness to JA, and was found to be similar to hy1 atmyc2 double mutants. These results suggest that MYC2 works downstream to HY1 in JA-induced expression of VSP2 gene.

\section{Overlapping functions of HY1 and MYC2 in Arabidopsis seedling development}

The atmyc2 mutants display hypersensitive response to $\mathrm{BL}$, and are epistatic to cry 1 and cry 2 [29]. In order to determine how these two light signaling components, HY1 and MYC2, genetically interact to control early seedling development, we measured the hypocotyl length of atmyc 2 hy 1 double mutants in various light conditions. Similar to hy1 or atmyc 2 single mutants, hy 1 atmyc2 double mutants did not show any altered growth in the dark. However, under WL conditions, hy1 atmyc2 double mutants displayed hypocotyl length similar to hy1 single mutants (Figure 6A and 6B). Furthermore, as shown in Figure $6 \mathrm{~A}$ and $6 \mathrm{C}$ to $6 \mathrm{E}$, hy1 atmyc 2 double mutants displayed hypocotyl length similar to hyl single mutants in $\mathrm{RL}, \mathrm{FR}$ and $\mathrm{BL}$ conditions. These results indicate that although additional mutation in $M Y C 2$ does not affect the phenotype of hy1 mutants in RL and FR, it is able to suppress the atmyc2 phenotype in BL. MYC2 acts as a negative regulator of light induced gene expression such as $C A B 1$ and $R B C S-1 A$. We examined how the additional mutation in $M Y C 2$ affects the expression of light-inducible genes in hy1 mutant background. The real time PCR analysis revealed that the expression of $C A B 1$ and $R C B S$ $1 A$ was similar to that of hy 1 single mutant in hy 1 atmyc2 background (Figure 6F and 6G). These results suggest that $H Y 1$ is epistatic to $M Y C 2$ in controlling the light induced gene expression.

\section{Discussion}

Although many components of light signaling pathways are known, the interconnections of these components in Arabidopsis seedling development is unclear. Moreover, very little information is available on cross talks of various components of light signaling with other signaling cascades and vice versa. In this study, we have demonstrated the genetic interactions of $H Y 1$ with two other light-signaling components, HY5 and MYC2, which 


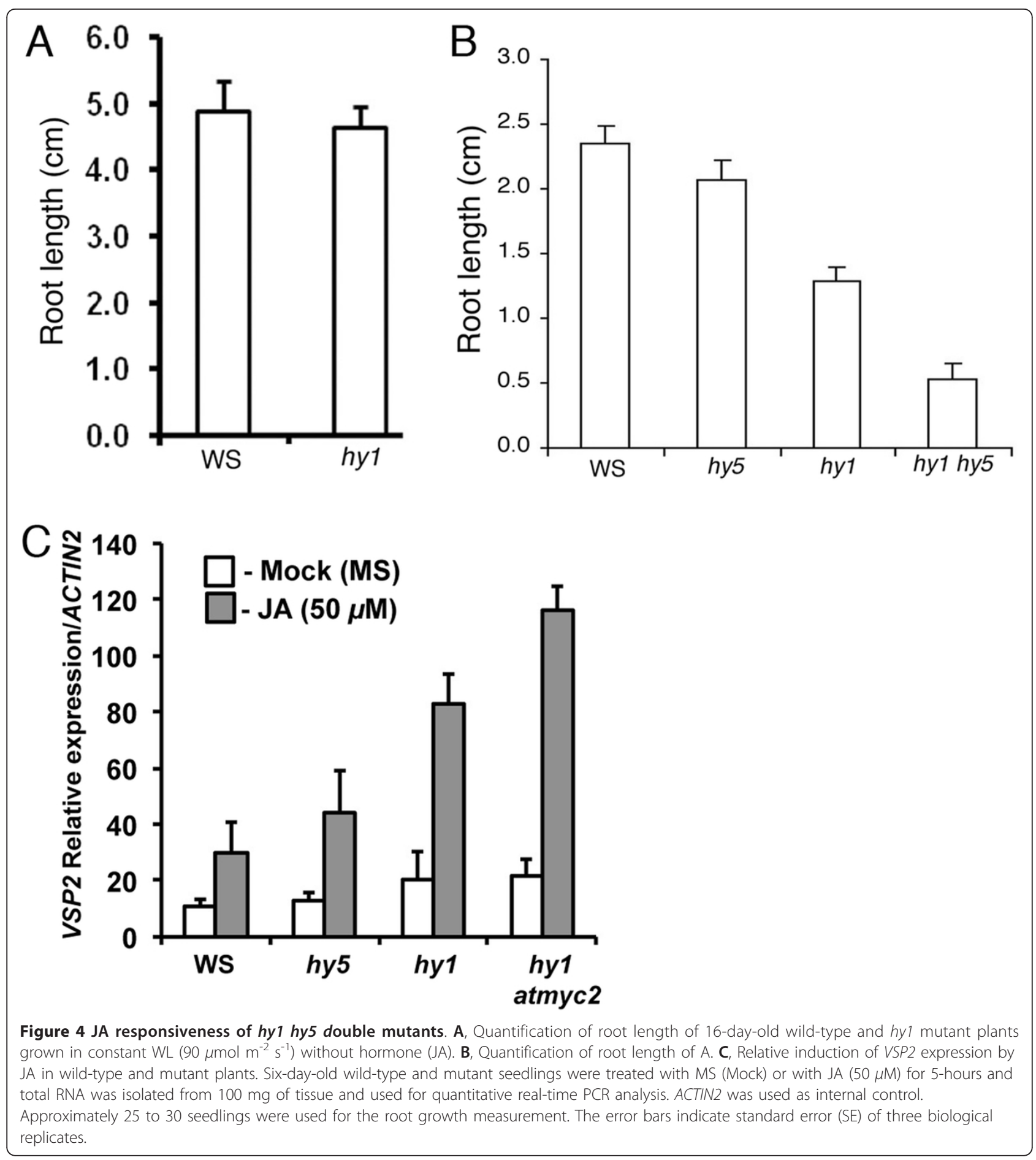

belong to two important families of transcription factors, bZIP and bHLH, respectively, in Arabidopsis seedling development. Furthermore, this study reveals that HY1, HY5 and MYC2 are functionally connected in JA signaling pathways.

An attempt to identify new genes that might enhance hy5 phenotype, similar to $C A M 7 / Z B F 3$ led to the identification of EHY5 [32]. Map based cloning and genetic complementation of ehy5 mutants reveal that EHY5 codes for HY1 (HO1), a rate-limiting enzyme that catalyzes the conversion of heme to biliverdin IX $\alpha$ (BV) in the chromophore biosynthesis pathway [54]. Phenotypic analyses under various light conditions have revealed that HY1 and HY5 function in an additive manner 


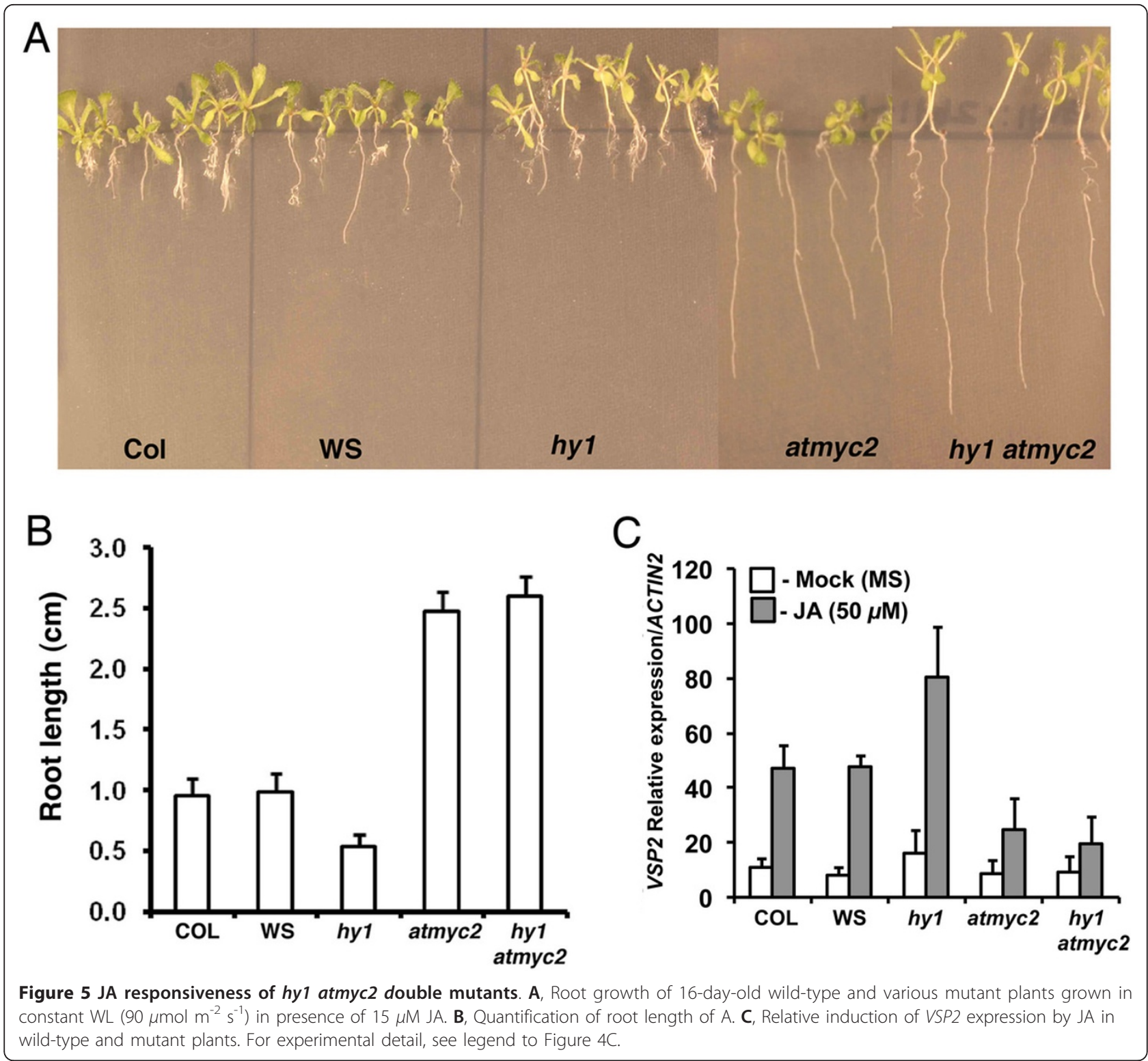

resulting in a super tall phenotype in WL. Similar additive function of HY5 and HY1 was also observed in the regulation of hypocotyl growth in FR. Genetic interaction studies between $H Y 1$ and $H Y 5$ reveal that they are likely to work in the same branched pathways of light signaling. On the other hand, mutations in $H Y 1$ does not affect the hy5 phenotype in BL. However, the additional mutation in $H Y 1$ is able to suppress the atmyc2 phenotype in BL. These results strongly suggest the wavelength specific interdependent functions of HY1, HY5 and MYC2 in the regulation of hypocotyl growth in Arabidopsis seedling development.

The expression of light regulated genes is down-regulated in hy1 mutant background. HY5 directly binds to the G-box present in the promoters of light regulated genes and promote their expression [39]. MYC2/ZBF1 also interacts with the Z-/G-box LRE present in the light-inducible promoters such as CAB1 and RBCS1A, however down-regulates their expression $[29,33,45]$. Analysis of light-regulated gene expression in hy1 hy5 double mutants reveal that HY1 and HY5 function in an additive manner and elevate the expression of light regulated genes. These two proteins also function in an additive manner to regulate the accumulation of chlorophyll and anthocyanin. On the other hand, the expression of $C A B 1$ and $R B C S-1 A$ in hy 1 atmyc 2 double mutant seedlings was similar to that of hyl single mutants, and thus suggesting that $\mathrm{HY} 1$ works downstream to $\mathrm{MYC} 2$ in the regulation of $C A B 1$ and $R B C S-1 A$ expression. It has been shown earlier that although atmyc 2 works downstream 


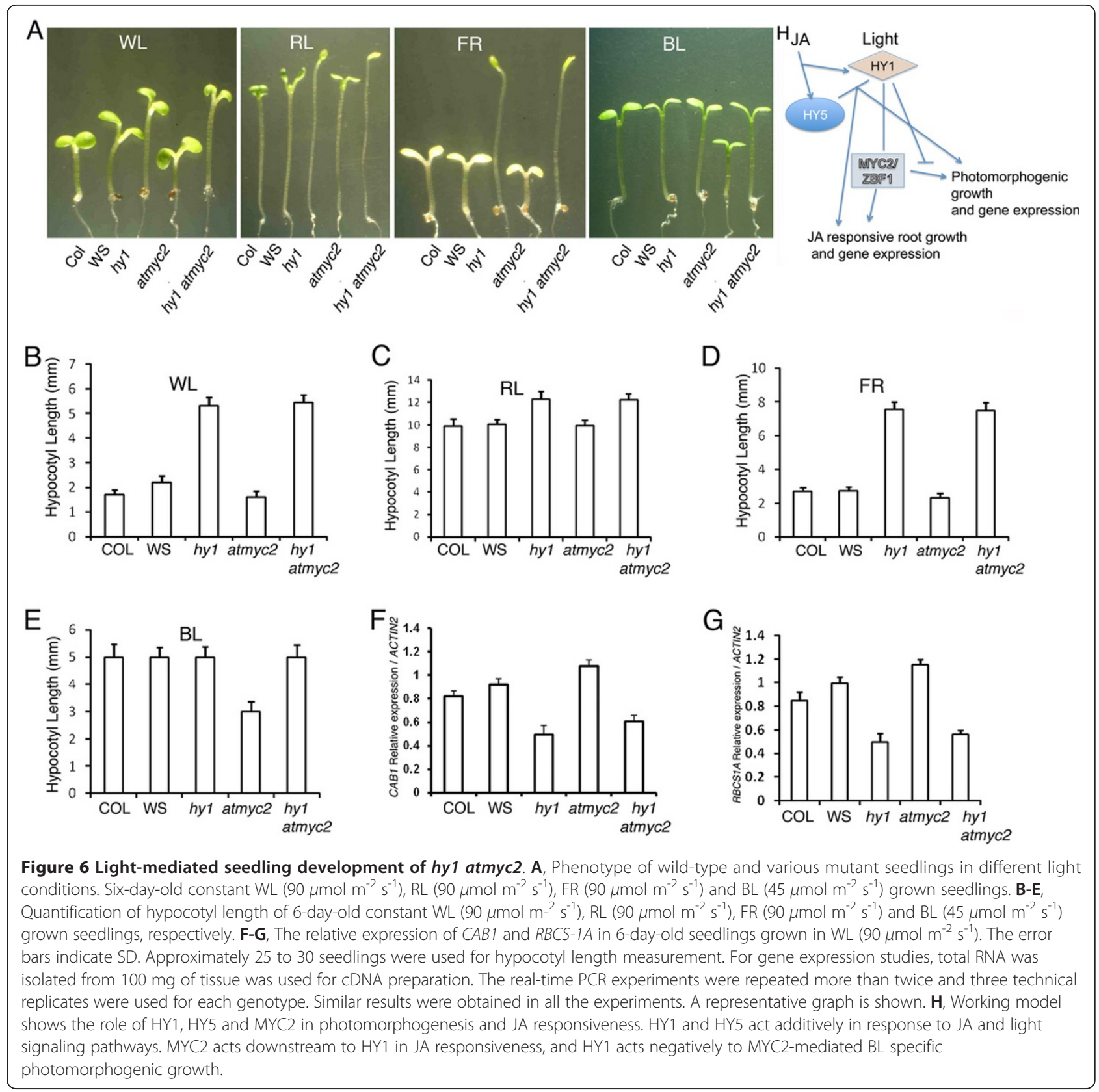

to cry1 and cry2 photoreceptors, phyA is epistatic to atmyc2 in BL [29].

Plant growth and development is a complex phenomenon, which is likely to be regulated through interactions between light and phytohormone signaling pathways. Recent studies have shown that signals from light and multiple hormonal signaling pathways cross talk through common downstream regulatory proteins such as MYC2 and HY5 [29,34-36,42-44,52,55]. For example, seedlings mutant for HY5 show altered balance of auxin and cytokinin signaling and also has decreased expression of two negative regulators of auxin signaling pathways such as
AXR2/IAA7 and SLR/IAA14. The functional overlap of light and JA signaling in defence, wound and shade response has been reported [56,57]. MYC2 regulates JA responses via differential regulation of an intermediate spectrum of transcription factors with activating or repressing roles. Furthermore, a JA activated MKK3MPK6 pathway negatively regulates the expression of $M Y C 2$ [53]. It has been shown that phytochorme deficient hy1 mutant seedlings overproduce JA and also display constant expression of JA inducible defense related genes such VSP1. The possible reason may be that there is reduction in the total photoactive phytochrome pool in 
the hy 1 mutant background and thereby resulting an altered light sensitivity. This may lead to photo-oxidative stress resulting in upregulation of JA synthesis in hy1 mutants [50]. The cross talks among multiple signaling pathways occur at the level of intermediate components of the signaling pathways rather than at the receptor level. For example, cross talks between light and JA signaling is mediated by the transcription factor (intermediate component) MYC2/ZBF1. MYC2/ZBF1 works in cryptochrome mediated blue light signaling pathways [29], however cry $1 /$ cry 2 mutants do not have altered JA responses.

It is worth mentioning here that it has earlier been reported that hy1 mutants display shorter roots than wild type plants [50]. However, this study does not find such difference in the absence of JA. The apparent discrepancy may be attributed to the developmental stages the observations were made. Whereas Zhai et al., 2007 found the difference at the early seedling stage, this study demonstrates the results of 16-day-old young adult plants, where the altered hypocotyl length was fairly maintained. In this study, our results demonstrate that HY5 and HY1 act additively or synergistically to regulate the JA-induced root-growth-inhibition and expression of JA-responsive genes. Although hy5 mutants do not show altered root growth in JA, the JA inducible gene VSP2 was upregulated in hy5 mutants in the presence of JA. These results indicate a negative regulatory role of HY5 in JA-mediated regulation of VSP2. On the other hand, MYC2 which acts as a negative regulator of light signaling, acts as a positive regulator of JA-mediated VSP2 expression Figure 5; [35]. Thus, both these transcription factors work in an opposite manner in light and JA signaling pathways.

\section{Conclusions}

This study demonstrates an overlapping function of HY1 with two important transcription factors of light signaling, HY5 and MYC2, in light and JA signaling pathways. The findings in this work will help to better understand the light signaling in Arabidopsis, and the cross talk of light and JA signaling pathways.

\section{Methods}

\section{Plant materials, growth conditions and generation of} double mutants

Arabidopsis (Arabidopsis thaliana) seeds were surface sterilized and sown on Murashige and Skoog plates, then kept at $4^{\circ} \mathrm{C}$ in darkness for 3 to 5 days, and transferred to specific light conditions at $22^{\circ} \mathrm{C}$. The intensities of $\mathrm{WL}$ and various colour lights (in the light-emitting diode chamber, Q-Beam 3200-A; (Quantum Devices)) used were described in Yadav et al. (2002). For the generation of double mutants such as hy1 atmyc2, homozygous hy1 (WS) mutant plants were genetically crossed with atmyc2-1
(Col-0) homozygous mutant lines. In the $\mathrm{F}_{2}$ generation, seedlings were grown in WL $\left(90 \mu \mathrm{mol} \mathrm{m}^{-2} \mathrm{~s}^{-1}\right)$ for the identification of hyl homozygous lines, and long hypocotyl hy1 mutants were selected and transferred to soil. To determine the genotype of AtMYC2 locus, about 40 seedlings from each line were tested by genomic PCR. $\mathrm{F}_{3}$ progenies that were homozygous for atmyc2 mutant plants were further examined by RT-PCR and considered as hy 1 atmyc2 double mutants. For measurement of hypocotyl length, $\sim 30$ seedlings were used in each genotype. The hypocotyl length measurement was repeated more than twice with similar results.

\section{Mutant screen and map-based cloning}

Ethyl methanesulfonate (EMS)-mutagenized, hy5KS50 M2 seeds of Arabidopsis thaliana ecotype Wassilewskija (Ws) were grown on MS media under WL conditions and hypocotyl length was compared with that of wild type and hy5KS50 mutant lines. Seedlings that showed enhanced and elongated hypocotyl length (as compared to hy5KS50) under all light conditions tested were selected and used for further studies. To identify the genetic basis of the EMS mutation in ehy5, we isolated the new ehy5 mutant, from the hy5KS50 ehy5 double mutant background. The double mutant plants were back-crossed successively to wild-type (WS) and the segregated ehy5 mutant seedlings in the $\mathrm{F}_{2}$ generation was selected and used for further back-crosses with wild type (WS) for four generations (to purify the background mutations in the EMS treated hy5-ks50 mutant plants) before physiological and genetic analysis. The ehy 5 mutant was out-crossed with Wild-type (Col) ecotype, and the mapping population was selected from $\mathrm{F}_{2}$ generation. A total of 823 individual $\mathrm{F}_{2}$ plants showing the ehy5 phenotype were selected for genetic mapping. Genomic DNA was prepared using the protocol described by Edwards et al. (1991). Cleaved amplified polymorphic sequence (CAPS) and simple sequence length polymorphism (SSLP) markers between $\mathrm{Col}$ and Ws were used for mapping EHY5. For genetic complementation analysis, a genomic fragment containing the entire $H Y 1 /$ At2g26670 coding sequence along with its promoter was amplified from the wild-type (Ws) by PCR and the PCR product was digested with HindIII and SmaI and inserted into same sites of modified pBI121 binary vector. The construct obtained was then introduced into ehy5 (hy1) mutant plants using A. tumefaciens-mediated transformation. Transformants were selected based on their resistance to kanamycin.

\section{Root growth measurement}

Seeds were on MS media in vertical square plates and stratified at $4^{\circ} \mathrm{C}$ in dark conditions for 4 days to induce uniform germination. The plates were placed vertically 
in racks, and the seedlings were grown under constant white light conditions $\left(90 \mu \mathrm{mol} \mathrm{m} \mathrm{m}^{-2} \mathrm{~s}^{-1}\right)$ for 16 days. The root length of wild type, single and double mutants was measured. Approximately 25 to 30 seedlings were used for the root length measurement. The experiments were repeated for three times with similar results.

\section{Root growth response to methyl jasmonate}

Seeds of wild type and mutant plants were plated on MS with $15 \mu \mathrm{M}$ of methyl jasmonate (Sigma) in square plates, after four days of stratification in cold $\left(4^{\circ} \mathrm{C}\right)$, plates were placed vertically in racks, and the seedlings were grown under constant white light conditions $\left(90 \mu \mathrm{mol} \mathrm{m}^{-2} \mathrm{~s}^{-1}\right)$ for 16 days. The root length of wild type, single and double mutants was measured. For determining the VSP2 expression, six-day-old whitelight grown seedlings were mock (only MS solution) or JA treated (50 $\mu \mathrm{M}$ JA in MS solution) for 5 hour. After the time period, seedlings were washed with sterile milliQ, excess water was removed with the tissue paper and the tissue was harvested and snap freeze in liquid nitrogen and total RNA was extracted from $100 \mathrm{mg}$ of tissue, using the RNeasy plant mini kit (Quaigen), and cDNA were synthesized from total RNA using titan one-tube RT-PCR system (Roche Applied Science) following the manufacturer's instructions. Real-time PCR analysis of gene expression was carried out by using LightCycler-FastStart DNA Master-PLUS SYBR Green (Roche Applied Science) and was performed using StepOne Real-Time PCR system (ABI). $C_{T}$ values of VSP2 were normalized, relative to that of ACTIN2 (Internal control).

The following primers were used for the experiment

VSP2-FP: 5' GGCCTTGCATCTTTACCAAAAC 3'

VSP2-RP: 5' GTAGTAGAGTGGATTTGGGAGC 3'

ACTIN2-FP: 5' AAAGGCTTAAAAAGCTGGGG 3'

ACTIN2-RP: 5' GGGACTAAAACGCAAAACGA 3'

\section{Real-time PCR analysis}

Total RNA was extracted from $100 \mathrm{mg}$ of tissue, using the RNeasy plant mini kit (Quaigen), according to manufacturer's protocol. RT-AMV reverse transcriptase (Roche Applied Science) was used for both semi-quantitative RTPCR and CDNA synthesis. Real-time PCR analysis of gene expression was carried out by using LightCycler-FastStart DNA Master-PLUS SYBR Green (Roche Applied Science) and was performed using Step-one Real-Time PCR system $(\mathrm{ABI}) . \mathrm{C}_{\mathrm{T}}$ values of $C A B 1$ and $R B C S 1 A$ were normalised, relative to that of ACTIN2 (Internal control). Real-time PCR was repeated more than thrice and in each biological experiment three technical replicates were used.

The following primers were used for the experiment HY1-FP: 5' GTGTATCCCTCTTCTCTATTCC 3'
HY1-RP: 5' TCTGAATCCTAGGTCGAGG 3' CAB1- FP: 5' GTTAACAACAACGCATGGC 3' CAB1-RP: 5' CCTCTCACACTCACGAAGCA 3' RBCS1A-FP: 5' TCGGATTCTCAACTGTCTGATG 3' RBCS1A-RP: 5' ATTTGTAGCCGCATTGTCCT 3' ACTIN2-FP: 5' TGATGCACTTGTGTGTGACAA 3' ACTIN2-RP: 5' GGGACTAAAACGCAAAACGA 3'

\section{Chlorophyll and anthocyanin measurements}

Chlorophyll and anthocyanin contents were measured following essentially the same protocols as described in [41]. For chlorophyll and anthocyanin estimation, 50 seedlings were used in each genotype and the experiment was repeated thrice; and in each biological experiment, four technical replicated were used.

\section{Acknowledgements}

This work is financially supported by Department of Biotechnology, Government of India to S.C and A.N.; V.B.R.P is a recipient of UGC fellowship, Government of India.

\section{Author details}

${ }^{1}$ National Institute of Plant Genome Research, New Delhi, India. ${ }^{2}$ School of Life Sciences, Jawharlal Neheru University, New Delhi, India. ${ }^{3}$ Department of Biotechnology, National Institute of Technology, Mahatma Gandhi Avenue, Durgapur 713209, West Bengal, India.

\section{Authors' contributions}

VBRP was involved in map-based cloning of EHY1/HY1, generation of double mutants, phenotypic characterization, JA responsiveness and gene expression study. VSK carried out the hy5 enhancer screen and identified and partly characterize the ehy5 mutant. AN helped in the map-based cloning and participated in the design of the manuscript. SC conceived of the study, participated in its design and coordination, and helped to draft the manuscript. All authors read and approved the final manuscript.

Received: 3 October 2011 Accepted: 17 March 2012

Published: 17 March 2012

\section{References}

1. Smith H: Phytochromes and light signal perception by plants-an emerging synthesis. Nature 2002, 407:585-591.

2. Sullivan JA, Deng XW: From seed to seed: The role of photoreceptors in Arabidopsi development. Dev Biol 2003, 260:289-297.

3. Cashmore AR, Jarillo JA, Wu YJ, Liu D: Cryptochromes: Blue light receptors for plants and animals. Science 1999, 284:760-765.

4. Briggs WR, Christie JM: Phototropins1 and 2: versatile plant blue light receptors. Trends Plant Sci 2002, 7:204-210.

5. Quail PH: Phytochrome photosensory signalling networks. Nat Rev Mol Cell Biol 2002, 3:85-93.

6. Lin C, Shalitin D: Cryptochrome structure and signal transduction. Annu Rev Plant Biol 2003, 2003(54):469-496.

7. $\mathrm{Li} \mathrm{QH}$, Yang $\mathrm{HQ}$ : Cryptochrome signaling in plants. Photochem Photobiol 2007, 83:94-101.

8. Wagner D, Koloszvari M, Quail PH: Two small spatially distinct regions of phytochrome B are required for efficient signaling rates. Plant Cell 1996, 8:859-719.

9. Kim Jl, Bhoo SH, Han YJ, Zarate X, Furuya M, Song PS: The PAS2 domain is required for dimerization of phytochromeA. J Photochem Photobiol 2006, 178:115-121.

10. Ted C, Ahmed S, Matt M, Peng L, Michael F, Robert AS: Obligate Heterodimerization of Arabidopsi Phytochromes C and E and interaction with the PIF3 Basic Helix-Loop - Helix Transcription Factor. Plant Cell 2009, 21:786-799. 
11. Sang Y, Li QH, Rubio V, Zhang YC, Mao J, Deng XW, Yang HQ: N-terminal domain-mediated homodimerization is required for photoreceptor activity of Arabidopsi CRYPTOCHROME 1. Plant Cell 2005, 17:1569-1584.

12. Guo H, Yang H, Mockler TC, Lin C: Regulation of flowering time by Arabidopsi photoreceptors. Science 1998, 279:1360-1363.

13. Mas P, Devlin PF, Panda S, Kay SA: Functional interaction of phytochrome B and cryptochrome 2. Nature 2000, 408:207-211.

14. Nagy F, Schafer E: Phytochromes control photomorphogenesis by differentially regulated, interacting signaling pathways in higher plants. Annu Rev Plant Biol 2002, 53:329-355.

15. Chen $M$, Chory J, Frankhauser C: Light signal transduction in higher Plants. Ann Rev Genet 2004, 38:87-117.

16. Ma L, Li J, Qu L, Hager J, Chen Z, Zhao H, Deng XW: Light control of Arabidopsi development entails coordinated regulation of genome expression and cellular pathways. Plant Cell 2001, 13:2589-2607.

17. Tepperman JM, Zhu T, Chang HS, Wang X, Quail PH: Multiple transcription factors genes are early targets of phytochrome A signalling. Proc Natl Sci USA 2001, 98:9437-9442.

18. Jiao Y, Yang H, Ma L, Sun N, Yu H, Liu T, Gao Y, Gu H, Chen Z, Wada M: A Genome-Wide Analysis of Blue-Light Regulation of Arabidopsis Transcription Factor Gene Expression during Seedling Development. Plant Physiol 2003, 133:1480-1493.

19. Oyama T, Shimura Y, Okada K: The Arabidopsi HY5 gene encodes a bZIP protein that regulates stimulus-induced development of root and hypocotyl. Genes Dev 1997, 1997(11):2983-2995.

20. Hudson M, Ringli C, Boylan MT, Quail PH: The FAR1 locus encodes a novel nuclear protein specific to phytochrome A signaling. Genes Dev 1999, 13:2017-2027.

21. Ballesteros ML, Bolle C, Lois LM, Moore JM, Vielle-Calzada JP, Grossniklaus U, Chua NH: LAF1, a MYB transcription activator for phytochrome $A$ signaling. Genes Dev 2001, 15:2613-2625.

22. Wang H, Deng XW: Arabidopsi FHY3 defines a key phytochrome A signaling component directly interacting with its homologous partner FAR1. EMBO J 2002, 21:1339-1349.

23. Kim YM, Woo JC, Song PS, Soh MS: HFR1, a phytochrome A signaling component, acts in a separate pathway from HY5, downstream of COP1 in Arabidopsi. Plant J 2002, 30:711-719.

24. Yang KY, Kim YM, Lee S, Song PS, Soh MS: Overexpression of a mutant basic helix-loop-helix protein HFR1, HFR1-N105, activates a branch pathway of light signaling in Arabidopsis. Plant Physiol 2003, 2003(133):1630-1642.

25. Parks HD, Ok PL, Sik JK, Shik DC, Hyun HS, Gil HN: The Arabidopsi COG1 gene encodes a Dof domain transcription factor and negatively regulates phytochrome signaling. Plant J 2003, 34:161-171.

26. Ward JM, Cufr AC, Denzel AM, Neff MM: The Dof Transcription factor OBP3 modulates phytochrome and cryptochrome signaling in Arabidopsi. Plant Cell 2005, 17:475-485.

27. Jang IC, Yang SW, Yang JY, Chua NH: Independent and interdependent functions of LAF1 and HFR1 in phytochrome A signaling. Genes Dev 2007, 21:2100-2111.

28. Yadav V, Kundu S, Chattopadhyay D, Negi P, Wei N, Deng XW, Chattopadhyay S: Light regulated modulation of Z-box containing promoters by photoreceptors and downstream regulatory components, COP1 and HY5, in Arabidopsi. Plant J 2002, 31:741-753.

29. Yadav V, Mallappa C, Gangappa NS, Bhatia S, Chattopadhyay S: A basic Helix-loop-helix transcription factor in Arabidopsi, MYC2, acts as a repressor of blue light-mediated photomorphogenic growth. Plant Cell 2005, 17:1953-1966.

30. Mallappa C, Yadav V, Negi P, Chattopadhyay S: A basic leucine zipper transcription factor, G-box binding Factor 1, regulates blue lightmediated photomorphogenic growth in Arabidopsi. J Biol Chem 2006, 281:22190-22199.

31. Mallappa C, Singh A, Gangappa SN, Chattopadhyay S: GBF1, a Transcription Factor of Blue Light Signaling in Arabidopsis, is Degraded in the Dark by a Proteasome-mediated Pathway Independent of COP1 and SPA1. J Biol Chem 2008, 283:35772-35782.

32. Kushwaha R, Singh A, Chattopadhyay S: Calmodulin7 plays an important role as transcriptional regulator in Arabidopsi seedling development. Plant Cell 2008, 20:1747-1759.
33. Gangappa SN, Prasad VBR, Chattopadhyay S: Functional Interconnection of MYC2 and SPA1 in the Photomorphogenic Seedling Development of Arabidopsi. Plant Physiol 2010, 154:1210-1219.

34. Abe H, Urao T, Ito T, Seki M, Shinozaki K, Yamaguchi-Shinozaki K: Arabidopsi AtMYC2 (bHLH) and AtMYB2 (MYB) function as transcriptional activators in Abscisic acid signaling. Plant Cell 2003, 15:63-78.

35. Boter M, Ruiz-Rivero O, Abdeen A, Prat S: Conserved MYC transcription factors play a key role in jasmonate signaling both in tomato and Arabidopsi. Genes Dev 2004, 8:1577-1591.

36. Lorenzo O, Chico JM, Sanchez-Serrano JJ, Solano R: JASMONATEINSENSITIVE1 encodes a MYC transcription factor essential to discriminate between different jasmonate-regulated defense responses in Arabidopsi. Plant Cell 2004, 2004(16):1938-1950.

37. Dombrecht B, Xue GP, Sprague SJ, Krikegaard JA, Ross JJ, Reid JB, Fit GP, Sewelam N, Schenk PM, Manners JM, Kazan K: MYC2 differentially modulates diverse jasmonate-dependent functions in Arabidopsi. Plant Cell 2007, 19:2225-2245.

38. Koornneef M, Rolff E, Spruit CJP: Genetic control of light-inhibited hypocotyl elongation in Arabidopsis thalian. Z Pflanzenphysiol 1980, 100:147-160.

39. Chattopadhyay S, Ang LH, Puente P, Deng XW, Wei N: Arabidopsi bZIP protein HY5 directly interacts with light-responsive promoters in mediating light control of gene expression. Plant Cell 1998, 10:673-683.

40. Ang LH, Chattopadhyay S, Wei N, Oyama T, Okada K, Batschauer A, Deng XW: Molecular interaction between COP1 and HY5 defines a regulatory switch for light control of Arabidopsi development. Mol Cell 1998, 1:213-222.

41. Holm M, Ma LG, Qu L, Deng XW: Two interacting bZIP proteins are direct targets of COP1-mediated control of light-dependent gene expression in Arabidopsi. Genes Dev 2002, 16:1247-1259.

42. Cluis CP, Mouchel CF, Hardtke CS: The Arabidopsi transcription factor HY5 integrates light and hormone signaling pathways. Plant J 2004, 38:332-347.

43. Sibout R, Sukumar P, Hettiarachchi C, Holm M, Muday GK, Hardtke CS: Opposite root growth phenotypes of hy versus hy5 hy mutants correlate with increased constitutive auxin signaling. PLoS Genet 2006, 2:e202.

44. Vandenbussche F, Habricot Y, Condiff AS, Maldiney R, Straeten DD, Ahmad M: HY5 is a point of convergence between cryptochrome and cytokinin signaling pathways in Arabidopsis thalian. Plant J 2007, 49:428-441.

45. Lee J, He K, Stolc V, Lee H, Figueroa P, Gao Y, Tongprasit W, Zhao H, Lee I, Deng XW: Analysis of transcription factor HY5 genomic binding sites revealed its hierarchical role in light regulation of development. Plant Cell 2007, 19:731-749.

46. Emborg TJ, Walker JM, Noh B, Vierstra RD: Multiple heme oxygenase family members contribute to the biosynthesis of the phytochrome chromophore in Arabidopsi. Plant Physiol 2006, 140:856-868.

47. Terry MJ: Phytochrome chromophore-deficient mutants. Plant Cell Environ 1997, 20:740-745.

48. Muramoto T, Kohchi T, Yokota A, Hwang I, Goodman HM: The Arabidopsi photomorphogenetic mutant hy is deficient in phytochrome chromophore biosynthesis as a result of a mutation in a plastid heme oxygenase. Plant Cell 1999, 11:335-348.

49. Davis SJ, Kurepa J, Vierstra RD: The Arabidopsis thalian HY1 locus, required for phytochrome chromophore biosynthesis, encodes a protein related to heme oxygenases. Proc Natl Acad Sci USA 1999, 96:6541-6546.

50. Zhai Q, Li CB, Zheng W, Wu X, Zhao J, Zhou G, Jiang H, Sun J, Lou Y, Li C: Phytochrome chromophore deficiency leads to overproduction of jasmonic acid and elevated expression of jasmonate-responsive genes in Arabidopsi. Plant Cell Phy 2007, 48:1061-1071.

51. Lopez-Juez E, Jarvis RP, Takeuchi A, Page MA, Chory J: New Arabidopsis cu mutants suggest a close connection between plastid- and phytochrome regulation of nuclear gene expression. Plant Physio/ 1998, 1998(118):803-815.

52. Chini A, Fonseca S, Fernandez GO, Garcia-Casado G, Lopez-Vidriero I, Lozano FM, Ponce MR, Micol JL, Solano R: The JAZ family of repressors is the missing link in jasmonate signalling. Nature 2007, 448:666-671.

53. Takahashi F, Yoshida R, Ichimura K, Mizoguchi T, Seo S, Yonezawa M, Maruyama K, Yamaguchi-Shinisali Y, Shinozaki K: The mitogen-activated Protein kinase cascade MKK3-MPK6 is an important part of the 
jasmonate signal transduction pathway in Arabidopsi. Plant Cell 2007, 19:805-818.

54. Osterlund MT, Wei N, Deng XW: The roles of photoreceptor systems and the COP1-targeted destabilization of HY5 in light control of Arabidopsis seedling development. Plant Plysiology 2000, 124:1520-1524.

55. Anderson JP, Badruzsaufari E, Schenk PM, Manners JM, Desmond OJ, Ehlert C, Maclean DJ, Ebert PR, Kazan K: Antagonistic interaction between Abscisic acid and jasmonate-ethylene signaling pathways modulates defense gene expression and disease resistance in Arabidopsis. Plant Cell 2004, 16:3460-3479.

56. Kazan K, Manners JM: The interplay of light and jasmonate signaling during defence and development. J Expt Bot 2011, 62:4087-4100.

57. Robson F, Okamoto H, Patrick E, Harris S-R, Wasternack C, Brearley C, Turner JG: Jasmonate and phytochrome A signaling in Arabidopsis wound and shade responses are integrated through JAZ1 stability. Plant Cell 2010, 22:1143-1160.

doi:10.1186/1471-2229-12-37

Cite this article as: Prasad et al.: Functional interconnections of HY1 with MYC2 and HY5 in Arabidopsis seedling development. BMC Plant Biology 2012 12:37.

\section{Submit your next manuscript to BioMed Central} and take full advantage of:

- Convenient online submission

- Thorough peer review

- No space constraints or color figure charges

- Immediate publication on acceptance

- Inclusion in PubMed, CAS, Scopus and Google Scholar

- Research which is freely available for redistribution

Submit your manuscript at www.biomedcentral.com/submit 